\title{
Nota
}

\section{DISPONIBILIDADE DE ZINCO PARA MUDAS DE EUCALIPTO EM SOLOS DE CERRADO ${ }^{(1)}$}

\author{
Fernando Antonio Vieira Rodrigues ${ }^{(2)}$, Nairam Félix de Barros ${ }^{(3)}$, Júlio César Lima \\ Neves ${ }^{(3)}$, Víctor Hugo Alvarez V. ${ }^{(3)}$ \& Roberto Ferreira Novais ${ }^{(3)}$
}

\section{RESUMO}

No Brasil, com a expansão da silvicultura clonal, o plantio de materiais genéticos mais produtivos e exigentes nutricionalmente tem aumentado o aparecimento de sintomas de deficiência de micronutrientes, principalmente de $\mathrm{B}$, e, mais recentemente, de $\mathrm{Cu}$ e de $\mathrm{Zn}$, sobretudo em áreas de Cerrado. Este trabalho teve por objetivo avaliar três métodos de determinação da disponibilidade de Zn (Mehlich-1, Mehlich-3 e DTPA pH 7,3) para mudas de eucalipto cultivadas em casa de vegetação, em amostras de seis solos de Cerrado, bem como a influência de propriedades do solo na eficiência desses extratores. Os tratamentos foram arranjados no esquema fatorial $6 \times 5$, correspondendo a seis solos e cinco doses de $\mathrm{Zn}\left(0,2,5,5,10\right.$ e $\left.20 \mathrm{mg} \mathrm{dm}^{-3}\right)$, com quatro repetições, em blocos ao acaso. Os níveis críticos de Zn em solos de Cerrado, para o crescimento de mudas de eucalipto, são iguais ou inferiores a 0,23 e $0,05 \mathrm{mg} \mathrm{dm}^{-3}$, pelos extratores Mehlich-1 e DTPA, respectivamente. Os teores de $\mathrm{Zn}$ no solo pelos três extratores, assim como os teores e conteúdo na planta, aumentam com a elevação das doses adicionadas de Zn e são altamente correlacionados entre si. A avaliação da disponibilidade de Zn para mudas de eucalipto pode ser feita com qualquer um dos três extratores; entretanto, o Mehlich-1 é o mais recomendado, pela facilidade operacional deste método em relação aos outros e por ele já ser usado na grande maioria dos laboratórios do país.

Termos de indexação: extratores, Mehlich-1, Mehlich-3, DTPA, fertilização.

(1) Parte da Dissertação de Mestrado do primeiro autor apresentada ao Programa de Pós-Graduação em Solos e Nutrição de Plantas do Departamento de Solos da Universidade Federal de Viçosa. Recebido para publicação em 24 de fevereiro de 2011 e aprovado em 13 de abril de 2012.

(2) Doutorando do Programa de Pós-Graduação em Solos e Nutrição de Plantas da Universidade Federal de Viçosa/UFV. Av. P. H. Rolfs s/n, CEP 36571-000 Viçosa-MG. E-mail: fvrgaucho@hotmail.com

(3) Professores do Departamento de Solos, UFV. E-mails: nfbarros@ufv.br, julio_m2003@yahoo.com.br, vhav@ufv.br, rfnovais@ufv.br 


\title{
SUMMARY: ZINC AVAILABITY FOR EUCALYPT SEEDLINGS IN CERRADO SOILS
}

\begin{abstract}
In Brazil, along with the expansion of clonal forestry, the management of more productive and more nutrient-demanding genotypes has led to the appearance of micronutrient deficiency symptoms, mainly of boron (B), and more recently, of copper $(C u)$ and of zinc ( $\mathrm{Zn}$ ), especially in Cerrado areas. The purpose of this study was to evaluate three extraction solutions to determine $Z n$ availability (Mehlich-1, Mehlich-3 and DTPA pH 7.3) for eucalyptus seedlings grown in a greenhouse, on six Cerrado soil samples, and to assess the influence of soil properties on the efficiency of these extractors. The treatments were arranged in a randomized block design, with four replications, in a $6 x 5$ factorial scheme, representing six soils and five $\mathrm{Zn}$ doses $\left(0,2.5,5,10\right.$, and $\left.20 \mathrm{mg} \mathrm{dm}^{-3}\right)$. The critical levels of Zn in Cerrado soils for eucalypts seedlings, by the extractors Mehlich-1 and DTPA, are 0.23 and $0.05 \mathrm{mg} \mathrm{dm}^{-3}$, respectively. The levels of soil $\mathrm{Zn}$ by the three extractants and the plant levels and content increased with increasing $\mathrm{Zn}$ doses and were highly correlated. $Z n$ availability to eucalypt seedlings can be determined using any of the three extractors. However, Mehlich-1 is the most recommended. Firstly, due to the ease of handling compared to the other methods and, secondly, for being widely used in most routine analyses in laboratories of the country.
\end{abstract}

Index terms: extractors, Mehlich-1, Mehlich-3, DTPA, fertilization.

\section{INTRODUÇÃO}

No Brasil, com a expansão da silvicultura clonal e o plantio de materiais genéticos mais produtivos e exigentes nutricionalmente, tem aumentado o aparecimento de sintomas de deficiência de micronutrientes, sobretudo de B, e, mais recentemente, de $\mathrm{Cu}$ e de Zn, especialmente em áreas de Cerrado.

Embora amplamente utilizada para diagnosticar a disponibilidade de macronutrientes com métodos bem definidos, a análise química do solo para micronutrientes tem ainda uso limitado e padrões indefinidos na área florestal. Em se tratando de culturas agrícolas, diversos métodos de extração têm sido utilizados para quantificar os teores de micronutrientes catiônicos no solo, destacando-se o Mehlich-1 e o DTPA.

Favarin et al. (2007) concluíram que os extratores DTPA e Mehlich-1 apresentaram eficiência semelhante para avaliar a disponibilidade de $\mathrm{Zn}$ no solo para a cultura do café, independentemente da espécie estudada (C. arabica e C. canephora). Ao testar a eficiência dos extratores Mehlich-1 (M-1), Mehlich-3 (M-3) e DTPA para o milho, Menezes et al. (2010) mostraram a viabilidade da utilização deles para avaliação da disponibilidade de Zn no solo, na ausência e presença de calagem. Rodrigues et al. (2010) concluíram que a avaliação da disponibilidade de $\mathrm{Cu}$ para mudas de eucalipto pode ser feita com qualquer um dos três extratores (M-1, M-3 e DTPA). Sequeira et al. (2011), em estudo em condições de campo com os mesmos extratores, recomendam o M-1 como extrator para micronutrientes catiônicos para o eucalipto. Por outro lado, Fonseca et al. (2010), após testarem M-1, M-3, DTPA e HCl, em diferentes relações solo:extrator, indicaram serem todos ineficientes para prever a disponibilidade de micronutrientes catiônicos para o trigo, após aplicação superficial de calcário.

Este trabalho teve por objetivo avaliar três métodos de determinação da disponibilidade de Zn (Mehlich-1, Mehlich-3 e DTPA pH 7,3) para mudas de eucalipto cultivadas em casa de vegetação, em amostras de seis solos de Cerrado, bem como a influência de propriedades do solo na eficiência desses extratores.

\section{MATERIAL E MÉTODOS}

Com base no estudo realizado por Sequeira (2007), foram coletadas amostras de solos de áreas de plantios comerciais de eucalipto na região do Cerrado de Minas Gerais (Quadro 1).

A unidade experimental foi constituída por um vaso com capacidade de $1,8 \mathrm{dm}^{3}$ de solo com duas mudas clonais de eucalipto ( $E$. urophylla $\mathrm{x} E$. grandis). Os tratamentos foram arranjados no esquema fatorial 6 $\mathrm{x} 5$, correspondendo a seis solos e cinco doses de Zn, com quatro repetições, em blocos ao acaso. As doses de $\mathrm{Zn}$ foram de 0, 2,5, 5, 10 e $20 \mathrm{mg} \mathrm{dm}^{-3}$, na forma de $\mathrm{ZnCl}_{2} \cdot 2 \mathrm{H}_{2} \mathrm{O}$.

Além dos tratamentos com $\mathrm{Zn}$, cinco dias antes do transplantio, foi aplicada adubação básica, em $\mathrm{mg} \mathrm{dm}^{-3}$, constituída de: 100 de N, 300 de $\mathrm{P}, 165$ de $\mathrm{K}, 70$ de S, 0,15 de Mo, 2,0 de B, 4,0 de Cu e 4,0 de Mn (modificado de Alvarez V., 1974); para os solos com teores de Ca e $\mathrm{Mg}$ menores do que 1,0 e $0,5 \mathrm{cmol}_{\mathrm{c}} \mathrm{dm}^{-3}$, respectivamente, foram aplicados cloretos de $\mathrm{Ca}$ e de $\mathrm{Mg}$ para atingir esses teores, de modo que não se aumentasse o $\mathrm{pH}$ do solo. Para facilitar a homogeneização 
Quadro 1. Caracterização química e física dos seis solos utilizados no estudo

\begin{tabular}{|c|c|c|c|c|c|c|c|c|c|c|}
\hline Solo* & $\mathbf{p H}\left(\mathrm{H}_{2} \mathrm{O}\right)^{(1)}$ & $\mathbf{C a}^{2+(2)}$ & $\mathrm{Mg}^{2+(2)}$ & $\mathbf{A l}^{3+(2)}$ & $\mathbf{H}+\mathbf{A l}^{(\mathbf{3})}$ & $\mathbf{P}^{(4)}$ & $\mathbf{K}^{(4)}$ & $\mathbf{M O}^{(5)}$ & P-rem ${ }^{(6)}$ & $\operatorname{Argila}^{(7)}$ \\
\hline & & \multicolumn{4}{|c|}{$\mathrm{cmol}_{\mathrm{c}} \mathrm{dm}^{-3}$} & \multicolumn{2}{|c|}{$-\mathrm{mg} \mathrm{dm}^{-3}$} & dag $\mathrm{kg}^{-1}$ & $m g L^{-1}$ & $\%$ \\
\hline 1 & 4,82 & 0,20 & 0,08 & 0,75 & 5,00 & 64,8 & 14 & 2,05 & 37,3 & 20,1 \\
\hline 2 & 5,71 & 2,26 & 2,12 & 0,00 & 6,25 & 2,3 & 238 & 4,80 & 16,7 & 55,3 \\
\hline 3 & 5,49 & 0,91 & 1,05 & 0,24 & 5,98 & 0,6 & 92 & 3,30 & 13,9 & 60,7 \\
\hline 4 & 4,66 & 0,20 & 0,08 & 0,94 & 7,95 & 0,9 & 17 & 3,87 & 22,3 & 42,6 \\
\hline 5 & 4,92 & 0,31 & 0,31 & 0,68 & 5,60 & 0,5 & 46 & 2,91 & 24,2 & 56,1 \\
\hline 6 & 5,06 & 0,63 & 0,14 & 0,80 & 4,05 & 1,0 & 51 & 2,02 & 35,3 & 35,0 \\
\hline
\end{tabular}

(1) $\mathrm{pH}$ em água, relação 1:2,5. ${ }^{(2)} \mathrm{Ca}^{2+}, \mathrm{Mg}^{2+} \mathrm{e} \mathrm{Al}^{3+}$ : extrator $\mathrm{KCl} 1 \mathrm{~mol} \mathrm{~L}^{-1}$. ${ }^{(3)} \mathrm{H}+\mathrm{AL}$ : extrator $\mathrm{Ca}(\mathrm{OAc})_{2} 0,5 \mathrm{~mol} \mathrm{~L}^{-1} \mathrm{pH}_{7,0 .}{ }^{(4)} \mathrm{P} \mathrm{e}$ K: extrator Mehlich-1. (5) MO (matéria orgânica): C. org. x 1,724 (Walkley-Black). (6) P-rem: fósforo remanescente (Alvarez V. et al., 2000). ${ }^{(7)}$ Argila (Ruiz, 2005).

* Origem dos solos: João Pinheiro (1), Vazante (2 e 3), Três Marias (4) e Curvelo (5 e 6).

com todo o volume de solo, os adubos foram dissolvidos em água. Em cobertura, aos 20 e 35 dias após o transplantio, foram aplicados $25 \mathrm{mg} \mathrm{dm}^{-3} \mathrm{de} \mathrm{N} \mathrm{e} 0,88$ $\mathrm{mg} \mathrm{dm}{ }^{-3}$ de Fe em cada adubação.

No momento do transplantio, as mudas, provenientes do enraizamento de miniestacas, tinham altura média em torno de $15 \mathrm{~cm}$. As raízes das mudas foram lavadas cuidadosamente com água, para remover porções do substrato eventualmente aderidas ao sistema radicular. Foi feita uma seleção das mudas por altura, separando-as em cada bloco. Os tratamentos foram aplicados em cada unidade experimental em separado. Durante o período do experimento, foi feito, semanalmente, o rodízio dos vasos.

Após a aplicação dos tratamentos e decorridos 15 dias de incubação, preparou-se a TFSA para análise dos teores de Zn pelos extratores Mehlich-1 (Mehlich, 1953), Mehlich-3 (Mehlich, 1984) e DTPA (Lindsay \& Norvell, 1978). A relação solo:extrator para o Mehlich-1 e Mehlich-3 foi de 1:10, com agitação a $200 \mathrm{rpm}$ por 5 min em agitador horizontal; os extratos foram pipetados após $16 \mathrm{~h}$ de repouso. Para o DTPA a relação foi de 1:2, com agitação por $2 \mathrm{~h}$ no mesmo agitador e rotação, sendo o extrato filtrado logo em seguida. Após 70 dias, as plantas foram cortadas e secas em estufa a $65{ }^{\circ} \mathrm{C}$ até atingirem peso constante. Posteriormente, foram pesadas, moídas e submetidas à digestão nítricoperclórica. Nos extratos obtidos (solo e planta), foram determinadas as concentrações de $\mathrm{Zn}$ por espectrofotometria de absorção atômica.

Os teores de $\mathrm{Zn}$ obtidos pelos três métodos, bem como os respectivos teores e conteúdos na matéria seca da parte aérea (MSPA) das plantas, foram submetidos à análise de variância. $\mathrm{O}$ efeito da aplicação de doses de Zn sobre a produção de MSPA, os teores recuperados pelos três extratores e os teores e conteúdos na MSPA foram avaliados por meio de análise de regressão. Os teores disponíveis no solo pelos três métodos foram correlacionados entre si. As taxas de recuperação pelos extratores foram correlacionadas com as características dos solos. Como suporte à execução das análises estatísticas, foram utilizados os softwares Statistica 6.0 e SAEG 5.0.

\section{RESULTADOS E DISCUSSÃo}

\section{Produção de matéria seca da parte aérea}

Para a maioria dos solos, não houve influência positiva das doses de Zn na produção de matéria seca da parte aérea (PMSPA) das mudas de eucalipto (Quadro 2). Nos solos 1 e 4 observou-se efeito da aplicação do nutriente, e a resposta foi negativa e linear, conforme os modelos, para o solo 1 ( $\left.\hat{y}=23,225-0,251^{*} x, \mathrm{R}^{2}=0,72\right)$ e para o solo $4\left(\hat{y}=26,022-0,092^{\text {ns }} x, R^{2}=0,87\right)$, em que PMSPA (g) ex = dose de Zn (md dm ${ }^{-3}$ ). Portanto, a disponibilidade natural de $\mathrm{Zn}$ nos solos estudados já era adequada ao pleno crescimento das mudas de eucalipto. Os menores teores estimados (declividade das retas do quadro 3 ) de Zn no solo pelo Mehlich-1 foi de $0,23 \mathrm{mg} \mathrm{dm}^{-3}$ (solo 6) e de $0,05 \mathrm{mg} \mathrm{dm}^{-3}$ (solo 4) pelo DTPA, para a condição de não adição de Zn. Estes teores são bem menores do que aqueles do limite superior da classe de disponibilidade média (nível crítico) usados em Minas Gerais e em São Paulo, ou seja, 1,5 $\mathrm{mg} \mathrm{dm}^{-3}$ de Zn pelo Mehlich-1 (Alvarez V. et al., 1999) e $1,2 \mathrm{mg} \mathrm{dm}^{-3}$ pelo DTPA (Raij et al., 1997), respectivamente.

Em estudo semelhante a este, Couto et al. (1985) não encontraram resposta em altura e PMSPA ao cultivarem mudas de eucalipto em casa de vegetação, em 12 solos de Cerrado, com baixos teores de $\mathrm{Zn}$, que receberam doses variando de 0 a $64 \mathrm{mg} \mathrm{dm}^{-3} \mathrm{de} \mathrm{Zn}$. Rodrigues et al. (2010) não encontraram influência de doses de $\mathrm{Cu}$ sobre a PMSPA de mudas de eucalipto nos mesmos seis solos usados neste trabalho.

Resultados de ausência de resposta de plantas de eucalipto à aplicação de micronutrientes, de modo geral, têm sido frequentemente observados em condições de umidade do solo elevada, próximo à capacidade de campo, em casa de vegetação (Novais 
et al., 1990). Isso tem ocorrido independentemente de origem do solo, textura, etc., mesmo para aqueles onde, no campo, os sintomas de sua deficiência são evidentes. As limitações por transporte de nutrientes até a raiz naquelas condições devido ao déficit hídrico e à manutenção de condições para intenso transporte (irrigação frequente) em casa de vegetação são, provavelmente, razões para a diferença de resultados (Novais et al., 1990).

Em casa de vegetação, mesmo que o teor do nutriente encontrado no solo seja considerado baixo ou, até mesmo, não detectável, ele pode ser suficiente para a planta crescer e desenvolver satisfatoriamente, não apresentando ganho de produção de matéria seca com sua aplicação.
De modo geral, uma das condições necessárias para obter ganhos na produção de matéria seca da parte aérea (PMSPA) é um aumento correspondente na quantidade absorvida de um determinado nutriente, que será constituinte da matéria seca produzida adicionalmente. Neste trabalho, como não houve aumento da PMSPA em resposta à aplicação de $\mathrm{Zn}$, o aumento dos teores e conteúdos de $\mathrm{Zn}$ na matéria seca da parte aérea (MSPA) ocorreu unicamente em resposta ao aumento na aquisição desse nutriente, como teoricamente era esperado. Portanto, em vez de trabalhar com a variável PMSPA, utilizaram-se teores e conteúdos de Zn na MSPA como indicativos de resposta positiva da planta à aplicação de Zn.

Quadro 2. Teores de Zn disponível pelo Mehlich-1, Mehlich-3 e DTPA, produção de matéria seca, teores e conteúdos de Zn na matéria seca de mudas de eucalipto cultivadas em amostras de seis solos de Cerrado (valores médios de quatro repetições), em função das doses de $\mathrm{Zn}$ aplicadas

\begin{tabular}{|c|c|c|c|c|c|c|c|}
\hline \multirow{2}{*}{ Solo } & \multirow{2}{*}{ Dose Zn } & \multicolumn{3}{|c|}{ Teor de Zn no solo } & \multirow{2}{*}{$\begin{array}{c}\text { Produção de } \\
\text { MSPA }\end{array}$} & \multirow{2}{*}{$\begin{array}{c}\text { Teor de Zn } \\
\text { na MSPA }\end{array}$} & \multirow{2}{*}{$\begin{array}{c}\text { Conteúdo de } \mathrm{Zn} \\
\text { na MSPA }\end{array}$} \\
\hline & & M-1 & M-3 & DTPA & & & \\
\hline & & & $\mathrm{mg} \mathrm{dm}$ & - & $\mathrm{g} /$ vaso $^{-1}$ & $\mathrm{mg} \mathrm{kg}{ }^{-1}$ & $\mu \mathrm{g} /$ vaso $^{-1}$ \\
\hline \multirow{5}{*}{1} & 0 & 0,56 & 0,30 & 0,28 & 25,02 & 14,39 & 359,2 \\
\hline & 2,5 & 2,73 & 2,14 & 1,83 & 21,78 & 25,96 & 561,8 \\
\hline & 5 & 4,80 & 4,19 & 3,67 & 20,60 & 37,34 & 767,1 \\
\hline & 10 & 8,82 & 7,94 & 7,20 & 20,63 & 55,32 & 1122,4 \\
\hline & 20 & 18,18 & 20,41 & 13,80 & 18,69 & 99,71 & 1875,5 \\
\hline \multirow{5}{*}{2} & 0 & 7,81 & 3,83 & 2,62 & 28,23 & 23,52 & 665,3 \\
\hline & 2,5 & 9,49 & 5,01 & 3,73 & 27,81 & 24,66 & 686,4 \\
\hline & 5 & 10,67 & 6,56 & 4,84 & 26,69 & 27,01 & 720,9 \\
\hline & 10 & 16,11 & 9,30 & 7,49 & 27,91 & 27,17 & 760,5 \\
\hline & 20 & 28,40 & 14,88 & 11,20 & 27,38 & 37,17 & 1019,3 \\
\hline \multirow{5}{*}{3} & 0 & 5,54 & 3,15 & 2,90 & 27,60 & 28,83 & 793,9 \\
\hline & 2,5 & 7,42 & 4,51 & 3,26 & 28,37 & 30,63 & 870,9 \\
\hline & 5 & 9,65 & 6,35 & 4,79 & 26,59 & 33,86 & 897,9 \\
\hline & 10 & 13,64 & 8,75 & 7,35 & 27,44 & 47,74 & 1317,6 \\
\hline & 20 & 23,94 & 16,01 & 11,85 & 27,17 & 57,86 & 1570,9 \\
\hline \multirow{5}{*}{4} & 0 & 0,53 & 0,26 & 0,35 & 26,26 & 12,75 & 334,9 \\
\hline & 2,5 & 2,61 & 1,86 & 1,77 & 25,51 & 19,67 & 501,3 \\
\hline & 5 & 4,94 & 3,48 & 3,56 & 25,34 & 26,87 & 683,5 \\
\hline & 10 & 8,28 & 6,82 & 7,25 & 25,45 & 39,73 & 1014,2 \\
\hline & 20 & 18,63 & 15,25 & 14,89 & 24,09 & 62,31 & 1512,9 \\
\hline \multirow{5}{*}{5} & 0 & 1,81 & 1,19 & 0,95 & 23,21 & 18,05 & 415,6 \\
\hline & 2,5 & 3,52 & 2,89 & 2,43 & 22,89 & 22,74 & 513,2 \\
\hline & 5 & 6,16 & 4,57 & 4,06 & 21,42 & 31,88 & 682,8 \\
\hline & 10 & 10,73 & 8,57 & 7,06 & 23,59 & 36,93 & 871,3 \\
\hline & 20 & 20,94 & 17,68 & 14,73 & 22,84 & 64,90 & 1481,0 \\
\hline \multirow{5}{*}{6} & 0 & 0,35 & 0,27 & 0,23 & 24,92 & 9,97 & 248,4 \\
\hline & 2,5 & 2,27 & 1,85 & 1,42 & 22,69 & 21,42 & 488,8 \\
\hline & 5 & 4,07 & 3,86 & 2,92 & 23,34 & 33,35 & 776,6 \\
\hline & 10 & 8,06 & 7,27 & 5,85 & 23,11 & 50,30 & 1160,2 \\
\hline & 20 & 16,21 & 11,49 & 10,87 & 23,24 & 93,18 & 2169,6 \\
\hline
\end{tabular}

Teor de Zn no solo: Mehlich-1 (M-1) e Mehlich-3 (M-3). Matéria seca da parte aérea (MSPA). 


\section{Teores de Zn disponível no solo}

Os teores de Zn pelo Mehlich-1, Mehlich-3 e DTPA foram linear e positivamente influenciados pelas doses aplicadas aos solos (Quadro 3). Contudo, a declividade das curvas variou com o solo, o que indica a influência das características dos solos na relação entre teores recuperados de Zn em função da dose aplicada (Quadro 3). Isso evidencia diferença quanto à capacidade-tampão de Zn dos solos e, também, quanto à influência da composição química de cada extrator, com reflexos no modo de extração e na quantidade extraída. As diferenças nos valores dos interceptos das equações que relacionam os teores com as doses adicionadas (Quadro 3) foram consequência da disponibilidade inicial de Zn no solo (Quadro 2). Aumentos lineares e positivos nos teores no solo em função de doses, com valores de $\mathrm{R}^{2}$ elevados, foram encontrados por Santos Neto (2003) ao trabalhar com solos de características contrastantes, em casa de vegetação.

As taxas de recuperação (média dos seis solos) de Zn pelos extratores Mehlich-1, Mehlich-3 eDTPA foram de 92,72 e $59 \%$ do Zn adicionado, respectivamente (Quadro 3). Santos Neto (2003) encontrou taxas de recuperação de 51,41 e $36 \%$ pelos extratores Mehlich1, Mehlich-3 e DTPA, respectivamente.

Considerando a ausência de resposta positiva das mudas às doses aplicadas, conclui-se que os teores de Zn disponível de 0,23 $\mathrm{mg} \mathrm{dm}^{-3}$ pelo Mehlich-1 e de $0,05 \mathrm{mg} \mathrm{dm}^{-3}$ de Zn pelo DTPA (Quadro 3), e talvez até menores do que estes, são suficientes para a produção de mudas de eucalipto produzidas em vasos com solo de Cerrado. Para o Mehlich-3, os menores teores estimados (intercepto) para os solos 1 e 4 foram negativos devido aos baixos teores naturais encontrados nesses solos, que foram de 0,30 e 0,26 $\mathrm{mg} \mathrm{dm}{ }^{-3} \mathrm{de} \mathrm{Zn}$, respectivamente (Quadro 2 ).

Os extratores ácidos apresentam maior poder de extração em virtude de sua dissolução ácida, o que causa a extração de frações que não são prontamente disponíveis para as plantas - por exemplo, dos óxidos (Cancela et al., 2001; Ferreira, 2003). Muitas plantas, a exemplo do eucalipto, apresentam acidificação intensa de sua rizosfera, e esse fenômeno pode disponibilizar às plantas frações não disponíveis no momento da análise do solo (Novais \& Smyth, 1999).

Em trabalhos como este - que consistem da aplicação de doses de nutrientes ao solo e extração química poucos dias após a aplicação - a interação do nutriente com a matriz sólida do solo é pequena, $\mathrm{e}$ estreita relação é encontrada entre os teores medidos pelos extratores e as respostas da planta - fato também constatado por Couto et al. (1985) ao trabalharem com doses de Zn. Entretanto, interações mais fortes poderão ocorrer com o passar do tempo, e aquelas boas relações encontradas inicialmente poderão desaparecer. Fato semelhante ocorre com o $\mathrm{P}$, onde ligações adicionais são formadas com o tempo, reduzindo a disponibilidade para a planta (Novais \& Smyth, 1999).

Os teores de $\mathrm{Zn}$ pelos três métodos de extração apresentaram alta correlação entre si, o que permite ajustar modelos preditivos (teor extrator $\mathrm{i}=\mathrm{f}$ (teor extrator i+1)) para estimar os teores de $\mathrm{Zn}$ para dois dos extratores a partir de um teor conhecido (M-1, M-3 ou DTPA), conforme os modelos a seguir: (M-3 $=0,282+$ $\left.0,694^{* * *} \mathrm{M}-1, \mathrm{R}^{2}=0,86\right)$; (M-3 = $-0,052+1,2244^{* * *}$ DTPA, $\left.\mathrm{R}^{2}=0,96\right) ; \mathrm{e}\left(\mathrm{DTPA}=0,480+0,5444^{* * *} \mathrm{M}-1, \mathrm{R}^{2}=0,83\right)$.

Correlações altamente significativas entre Mehlich-3 e DTPA na extração de Zn $(r=0,78)$ foram obtidas em um estudo com amostras de 29 solos ácidos da Espanha (Obrador et al., 2007). Sequeira (2007), estudando teores de $\mathrm{Zn}$ em 150 amostras de solos em campo, encontrou coeficientes de correlação linear de 0,94 entre Mehlich-1 e Mehlich-3, de 0,95 entre Mehlich-1 e DTPA e de 0,90 entre Mehlich-3 e DTPA.

\section{Relação entre Zn na parte aérea das mudas e doses aplicadas}

As relações entre os teores e conteúdos de $\mathrm{Zn}$ na matéria seca da parte aérea (MSPA) em função das doses de $\mathrm{Zn}$ aplicadas foram lineares (Quadro 4). Os teores estimados pelas equações na maior dose de $\mathrm{Zn}$ testada $\left(20 \mathrm{mg} \mathrm{dm}^{-3}\right)$ foram de 99,$3 ; 36,2 ; 59,2 ; 63,1$;

Quadro 3. Equações para teores de Zn disponível (y, $\left.\mathrm{mg} \mathrm{dm}^{-3}\right)$ pelo Mehlich-1, Mehlich-3 e DTPA em função das doses de $\mathrm{Zn}\left(\mathrm{x}, \mathrm{mg} \mathrm{dm}^{-3}\right)$ adicionadas a amostras de seis solos de Cerrado

\begin{tabular}{|c|c|c|c|c|c|c|}
\hline \multirow{2}{*}{ Solo } & \multicolumn{2}{|l|}{ Mehlich-1 } & \multicolumn{2}{|l|}{ Mehlich-3 } & \multicolumn{2}{|c|}{ DTPA pH 7,3 } \\
\hline & Equação & $\mathbf{R}^{2}$ & Equação & $\mathbf{R}^{2}$ & Equação & $\mathbf{R}^{2}$ \\
\hline 1 & $\hat{\mathrm{y}}=0,43+0,878^{* * *} \mathrm{x}$ & 0,99 & $\hat{\mathrm{y}}=-0,56+1,006^{* * *} \mathrm{x}$ & 0,98 & $\hat{\mathrm{y}}=0,25+0,680^{* * *} \mathrm{x}$ & 0,99 \\
\hline 2 & $\hat{\mathrm{y}}=6,61+1,051^{* * *} \mathrm{x}$ & 0,98 & $\hat{\mathrm{y}}=3,74+0,556^{* * *} \mathrm{x}$ & 0,99 & $\hat{\mathrm{y}}=2,73+0,433^{* * *} \mathrm{x}$ & 0,99 \\
\hline 3 & $\hat{\mathrm{y}}=5,12+0,922^{* * *} \mathrm{x}$ & 0,99 & $\hat{\mathrm{y}}=2,95+0,640^{* * * *} \mathrm{x}$ & 0,99 & $\hat{\mathrm{y}}=2,53+0,466^{* * * *} \mathrm{x}$ & 0,99 \\
\hline 4 & $\hat{\mathrm{y}}=0,27+0,897^{* * *} \mathrm{x}$ & 0,99 & $\hat{\mathrm{y}}=-0,10+0,751^{* * *} \mathrm{x}$ & 0,99 & $\hat{\mathrm{y}}=0,05+0,735^{* * *} \mathrm{x}$ & 0,99 \\
\hline 5 & $\hat{\mathrm{y}}=1,37+0,968^{* * *} \mathrm{x}$ & 0,99 & $\hat{\mathrm{y}}=0,75+0,831^{* * *} \mathrm{x}$ & 0,99 & $\hat{\mathrm{y}}=0,67+0,690^{* * *} \mathrm{x}$ & 0,99 \\
\hline 6 & $\hat{\mathrm{y}}=0,23+0,794^{* * *} \mathrm{x}$ & 0,99 & $\hat{\mathrm{y}}=0,72+0,563^{* * *} \mathrm{x}$ & 0,98 & $\hat{\mathrm{y}}=0,23+0,538^{* * *} \mathrm{x}$ & 0,99 \\
\hline
\end{tabular}

***: significativo a $0,1 \%$ pelo teste $\mathrm{F}$. 
Quadro 4. Equações de regressão para os teores (y, $\left.\mathrm{mg} \mathrm{kg}^{-1}\right)$ e conteúdos (y, ug vaso-1) de Zn na matéria seca da parte aérea de plantas de eucalipto em função das doses $\left(x, \mathrm{mg} \mathrm{dm}^{-3}\right)$ de $\mathrm{Zn}$ adicionadas a amostras de seis solos de Cerrado

\begin{tabular}{|c|c|c|c|c|c|}
\hline \multirow{2}{*}{ Solo } & \multicolumn{2}{|l|}{ Teor } & \multirow{2}{*}{$\hat{\mathrm{y}}>$} & \multicolumn{2}{|l|}{ Conteúdo } \\
\hline & Equação & $\mathbf{R}^{2}$ & & Equação & $\mathbf{R}^{2}$ \\
\hline 1 & $\hat{\mathrm{y}}=14,92+4,219 * * * \mathrm{x}$ & 0,99 & 99,3 & $\hat{\mathrm{y}}=372,3+75,315^{* *} \mathrm{x}$ & 0,99 \\
\hline 2 & $\hat{\mathrm{y}}=22,95+0,661^{*} \mathrm{x}$ & 0,94 & 36,2 & $\hat{\mathrm{y}}=637,9+17,675^{*} \mathrm{x}$ & 0,95 \\
\hline 3 & $\hat{\mathrm{y}}=28,13+1,554^{* *} \mathrm{x}$ & 0,96 & 59,2 & $\hat{\mathrm{y}}=779,0+41,505^{* *} \mathrm{x}$ & 0,95 \\
\hline 4 & $\hat{\mathrm{y}}=13,75+2,468^{* *} \mathrm{x}$ & 0,99 & 63,1 & $\hat{\mathrm{y}}=367,8+58,875^{* *} \mathrm{x}$ & 0,99 \\
\hline 5 & $\hat{\mathrm{y}}=17,66+2,299 * * \mathrm{x}$ & 0,98 & 63,6 & $\hat{\mathrm{y}}=393,7+53,203^{* *} \mathrm{x}$ & 0,99 \\
\hline 6 & $\hat{\mathrm{y}}=10,89+4,101^{* * *} \mathrm{x}$ & 0,99 & 92,9 & $\hat{\mathrm{y}}=255,6+95,088^{* * *} \mathrm{x}$ & 0,99 \\
\hline
\end{tabular}

${ }^{*},{ }^{* *} \mathrm{e}^{* * *}$ : significativo a 5,1 e $0,1 \%$ pelo teste $\mathrm{F}$, respectivamente. $\hat{\mathrm{y}}>$ : teor estimado para a dose maior $\left(20 \mathrm{mg} \mathrm{dm}^{-3}\right)$

63,6; e 92,9 mg kg-1 para os solos 1, 2, 3, 4, 5 e 6 , respectivamente (Quadro 4). O solo 2 possui maior teor de matéria orgânica (MO), e as plantas nele cultivadas apresentaram menor teor de Zn na MSPA, indicando ser a MO uma característica que tampona esse nutriente no solo, restringindo sua absorção pela planta. Por outro lado, os solos 1 e 6 possuem os menores teores de argila e os maiores valores de Prem, e neles as plantas apresentaram teores de $\mathrm{Zn}$ mais elevados na MSPA, devido à menor capacidadetampão desses solos.

Para diversas culturas, como arroz, cevada, milho, soja e trigo, os valores das correlações lineares entre teores foliares e teores disponíveis no solo têm sido bastante variáveis. Em geral, o extrator adequado para uma cultura não é, necessariamente, o melhor para outras, conforme relatado por vários autores (Cancela et al., 2001; Silva et al., 2003; Gonçalves Jr. et al., 2006; Obrador et al., 2007). Isso indica a necessidade de estudos para cada solo e cultura específica na seleção do extrator mais eficiente.

Portanto, diante dos dados obtidos e devido à similaridade dos valores de $\mathrm{R}^{2}$ dos dois modelos explicativos (teor no solo $=\mathrm{f}($ dose $)$ e conteúdo na planta $=\mathrm{f}($ dose $)$ e do modelo preditivo (teor extrator $\mathrm{i}=\mathrm{f}$ (teor extrator i+1)), a avaliação da disponibilidade de $\mathrm{Zn}$ para o eucalipto pode ser feita com qualquer um dos três extratores testados. Contudo, como o Mehlich-1 é o extrator utilizado nos laboratórios de análise de rotina em Minas Gerais e em vários Estados do país (Lopes \& Alvarez V., 1999), ele seria mais recomendado, pela facilidade operacional e por ser usado na grande maioria dos laboratórios brasileiros. Entretanto, como mencionado, a extração química poucos dias após a aplicação do nutriente pode não revelar o efeito da forte interação do nutriente com a matriz sólida do solo, e boas relações podem ser encontradas com os três extratores. Por outro lado, se as extrações forem feitas após um tempo maior de contato do nutriente com o solo, permitindo o estabelecimento de ligações adicionais, poderá haver redução da disponibilidade para a planta e, possivelmente, os extratores apresentarão eficiência diferenciada. Além disso, também é de se esperar que as taxas de recuperação sejam menores do que as encontradas neste trabalho quando se aumenta o tempo de contato do nutriente com o solo. Fato semelhante ocorre com o P (Novais \& Smyth, 1999).

\section{Relação entre teor natural de Zn no solo e Zn na planta}

De acordo com Bray (1948), o extrator idealé aquele que extrai as formas disponíveis para as plantas, em solos com diferentes características, que apresentam adequação aos laboratórios de rotina. Além disso, devese correlacionar estreitamente com as respostas das culturas à presença dos teores naturais dos nutrientes no solo. Na tentativa de atender a esses preceitos, foram estabelecidas relações comparando o comportamento dos três extratores, nas condições de não aplicação (dose zero) e aplicação dos nutrientes (demais doses testadas), conforme mostram os quadros 5 e 6 .

Os modelos ajustados que relacionam produção de matéria seca da parte aérea (PMSPA), teor e conteúdo na parte aérea com os teores naturais de $\mathrm{Zn}$ disponíveis (dose zero) (Quadro 5) foram semelhantes para todos os extratores quanto aos coeficientes de determinação. Os resultados do quadro 5 mostram aumento linear e positivo da PMSPA com o teor de $\mathrm{Zn}$ do solo, o que indica que não se atingiu a máxima PMSPA nem mesmo com os maiores teores naturais nos solos de $7,81,3,83$ e $2,90 \mathrm{mg} \mathrm{dm}^{-3}$ de $\mathrm{Zn}$, respectivamente pelo Mehlich-1, Mehlich-3 e DTPA (Quadro 2), sendo, portanto, os níveis críticos de $\mathrm{Zn}$ no solo maiores do que estes naturalmente encontrados. Esses resultados indicariam que o teor crítico de $\mathrm{Zn}$ não dependeria da capacidade-tampão do solo, contrariamente ao indicado em trabalhos anteriores e ao que se tem observado para P (Novais \& Smyth, 1999). Esses resultados contrastantes mostram a importância e a influência que o método pode ter nos resultados do estudo.

Os coeficientes de correlação entre os teores de $\mathrm{Zn}$ disponíveis no solo com os teores e conteúdos nas plantas foram mais elevados para todos os extratores 
na dose zero que nas demais doses (Quadro 6). As correlações na dose zero tiveram valores muito próximos para os três extratores; entretanto, nas demais doses, o Mehlich-1 apresentou valores menores que os de Mehlich-3 e DTPA.

\section{Características do solo e recuperação de Zn pelos extratores}

A correlação entre características do solo e disponibilidade de $\mathrm{Zn}$ indica a sensibilidade dos extratores à capacidade-tampão do solo (Quadro 7).

As correlações com os teores de MO e P-rem foram as que tiveram os maiores valores para o Mehlich-1 (M-1), indicando que este extrator extrai mais Zn de solos com maiores teores de $\mathrm{MO}$ e menor P-rem (Quadro 7), o que, à luz do conhecimento atual, não faz sentido, pois era de se esperar o contrário, ou seja, ao aumentar o teor de argila (diminui o P-rem), diminuiria a taxa de recuperação pelo extrator, pois o número de sítios de adsorção de Zn seria aumentado, como o encontrado para o Mehlich-3 (M-3) neste trabalho e por Couto et al. (1985) para os extratores $\mathrm{M}-1$ e $\mathrm{HCl} 0,1 \mathrm{~mol} \mathrm{~L}^{-1}$. Por outro lado, é razoável considerar que, mesmo que o desgaste do extrator seja maior nos solos mais argilosos e com maiores teores

Quadro 5. Equações de regressão entre a PMSPA, teores e conteúdos de Zn na MSPA de mudas de eucalipto cultivadas em amostras de seis solos de Cerrado, em função dos teores naturais no solo (dose zero) extraídos pelo Mehlich-1, Mehlich-3 e DTPA

\begin{tabular}{|c|c|c|c|c|c|c|}
\hline \multirow{2}{*}{ Solo } & \multicolumn{2}{|c|}{ PMSPA (g/vaso) } & \multicolumn{2}{|c|}{ Teor na planta $\left(\mathrm{mg} \mathrm{kg}^{-1}\right)$} & \multicolumn{2}{|c|}{ Conteúdo na planta ( $\mu g / v a s o)$} \\
\hline & Equação & $\mathbf{R}^{2}$ & Equação & $\mathbf{R}^{2}$ & Equação & $\mathbf{R}^{2}$ \\
\hline M-1 & $\hat{\mathrm{y}}=24,62+0,452^{*} \mathrm{x}$ & 0,58 & $\hat{\mathrm{y}}=8,8+7,21^{* * *} \mathrm{x}-0,67^{* *} \mathrm{x}^{2}$ & 0,97 & $\hat{\mathrm{y}}=214+182,3^{*} \mathrm{x}-15,62^{*} \mathrm{x}^{2}$ & 0,94 \\
\hline M-3 & $\hat{\mathrm{y}}=24,60+0,853^{*} \mathrm{x}$ & 0,53 & $\hat{\mathrm{y}}=8,9+11,82^{*} \mathrm{x}-1,99^{\circ} \mathrm{x}^{2}$ & 0,91 & $\hat{\mathrm{y}}=234+256,3^{\circ} \mathrm{x}-33,90+\mathrm{x}^{2}$ & 0,90 \\
\hline DTPA & $\hat{\mathrm{y}}=24,52+1,108^{*} \mathrm{x}$ & 0,53 & $\hat{\mathrm{y}}=11,1+5,58^{* *} \mathrm{x}$ & 0,92 & $\hat{\mathrm{y}}=262+169,8^{* * *} \mathrm{x}$ & 0,96 \\
\hline
\end{tabular}

ns $,+{ }^{\circ},{ }^{*},{ }^{* *} \mathrm{e}^{* * * *}$ : não significativo e significativo a $15,10,5,1$ e $0,1 \%$ pelo teste $\mathrm{F}$, respectivamente. As equações foram ajustadas com as médias de cada solo. PMSPA: produção de matéria seca da parte aérea; MSPA: matéria seca da parte aérea; M-1: Mehlich-1; M3: Mehlich-3.

Quadro 6. Coeficiente de correlação linear (r) entre teor e conteúdo de Zn na matéria seca da parte aérea de mudas de eucalipto cultivadas em amostras de seis solos de Cerrado e teor de Zn disponível pelo Mehlich1, Mehlich-3 e DTPA, na dose zero e nas demais doses

\begin{tabular}{|c|c|c|c|c|c|c|}
\hline \multirow[t]{2}{*}{ DOSE } & \multicolumn{3}{|c|}{ Teor disponível no solo com teor na planta } & \multicolumn{3}{|c|}{ Teor disponível no solo com conteúdo na planta } \\
\hline & Mehlich-1 & Mehlich-3 & DTPA & Mehlich-1 & Mehlich-3 & DTPA \\
\hline Zero & $0,81^{* * * *}$ & $0,87^{* * *}$ & $0,88^{* * * *}$ & $0,86^{* * *}$ & $0,91^{* * *}$ & $0,89^{* * * *}$ \\
\hline Demais $^{(1)}$ & $0,58^{* * * *}$ & $0,80^{* * * *}$ & $0,80^{* * * *}$ & $0,69^{* * *}$ & $0,82^{* * *}$ & $0,85^{* * *}$ \\
\hline
\end{tabular}

${ }^{(1)}$ Demais: doses de $2,5,5,10$ e $20 \mathrm{mg} \mathrm{dm}^{-3}$ de $\mathrm{Zn} .{ }^{* * *}$ : significativo a $0,1 \%$ pelo teste $\mathrm{F}$.

Quadro 7. Coeficientes de correlação linear (r) entre características do solo, teor e conteúdo de Zn na MSPA, e as declividades das equações do quadro 4 - Zn recuperado pelo Mehlich-1(M-1), Mehlich-3 (M-3) e DTPA, em função do adicionado às amostras de seis solos de Cerrado

\begin{tabular}{|c|c|c|c|c|c|}
\hline \multirow{2}{*}{ Atributos do solo } & \multicolumn{3}{|c|}{ Extratores } & \multicolumn{2}{|c|}{ Planta } \\
\hline & M-1 & M-3 & DTPA & Teor & Conteúdo \\
\hline $\mathrm{pH}$ & $0,54^{* * *}$ & $-0,69^{* * * *}$ & $-0,96^{* * *}$ & $-0,10^{\mathrm{ns}}$ & $0,04^{\mathrm{ns}}$ \\
\hline $\mathrm{H}+\mathrm{Al}$ & $0,49^{* *}$ & $0,02^{\mathrm{ns}}$ & $0,25^{\mathrm{ns}}$ & $-0,19^{\mathrm{ns}}$ & $-0,11^{\mathrm{ns}}$ \\
\hline Matéria orgânica & $0,82^{* * *}$ & $-0,44^{* *}$ & $-0,35^{\mathrm{ns}}$ & $-0,27^{\mathrm{ns}}$ & $-0,13^{\mathrm{ns}}$ \\
\hline P-rem & $-0,71^{* * *}$ & $0,47^{* *}$ & $0,46^{* *}$ & $0,20^{\mathrm{ns}}$ & $0,02^{\mathrm{ns}}$ \\
\hline Argila & $0,64^{* * *}$ & $-0,54^{* *}$ & $-0,46^{* *}$ & $-0,19^{\mathrm{ns}}$ & $-0,02^{\mathrm{ns}}$ \\
\hline
\end{tabular}

ns, ** $\mathrm{e}^{* * *}$ : não significativo e significativo a 1 e $0,1 \%$ pelo teste $\mathrm{F}$, respectivamente. 
Quadro 8. Equações de regressão para as taxas de recuperação (mg dm-3/mg dm-3) de $\mathrm{Zn}$ pelo $\mathrm{Mehlich}-1$, Mehlich-3 e DTPA, em função de algumas características de seis solos de Cerrado

\begin{tabular}{|c|c|c|c|c|c|}
\hline \multicolumn{2}{|l|}{ Mehlich-1 } & \multicolumn{2}{|l|}{ Mehlich-3 } & \multicolumn{2}{|l|}{ DTPA } \\
\hline Equação & $\mathbf{R}^{2}$ & Equação & $\mathbf{R}^{2}$ & Equação & $\mathbf{R}^{2}$ \\
\hline $\mathrm{TR}=0,71+0,0647^{*} \mathrm{MO}$ & 0,65 & $\mathrm{TR}=1,05-0,00645^{\circ} \mathrm{ARG}$ & 0,49 & $\mathrm{TR}=1,90-0,2519^{* *} \mathrm{pH}$ & 0,81 \\
\hline $\mathrm{TR}=1,08+0,0063^{\circ} \mathrm{P}-\mathrm{rem}$ & 0,49 & $\mathrm{TR}=1,99-0,2403^{\circ} \mathrm{pH}$ & 0,47 & $\mathrm{TR}=2,4-0,0049^{\circ}$ P-rem $-0,3216^{* *} \mathrm{pH}$ & 0,86 \\
\hline $\mathrm{TR}=0,76+0,0036^{\circ} \mathrm{ARG}$ & 0,41 & $\mathrm{TR}=0,51+0,010^{\circ} \mathrm{P}$-rem & 0,45 & - & - \\
\hline
\end{tabular}

${ }^{\circ},{ }^{*}, \mathrm{e}^{* * * *}:$ significativo a $10,5,1$ e $0,1 \%$ pelo teste $\mathrm{F}$, respectivamente. TR: taxa de recuperação; MO: matéria orgânica (dag kg-1); P-rem: fósforo remanescente $\left(\mathrm{mg} \mathrm{L}^{-1}\right)$; ARG: argila (\%).

de MO, a correlação com o Zn extraído permanecesse positiva, devido ao maior teor de $\mathrm{Zn}$ nesses solos, ou seja, o efeito fonte de Zn suplantaria o desgaste do extrator. O P-rem é uma característica que tem sido usada como medida do fator capacidade de $\mathrm{P}$ e de $\mathrm{S}$ (Alvarez V. et al., 2000).

Para o M-3, as melhores correlações foram com $\mathrm{pH}$, teor de argila e P-rem, o que significa maior extração de $\mathrm{Zn}$ em solos com valores menores de $\mathrm{pH}$ e argila e maiores de P-rem. O DTPA apresentou correlação estreita apenas com o pH, indicando ser o extrator mais sensível a diferentes condições de $\mathrm{pH}$, diminuindo a extração de $\mathrm{Zn}$ à medida que se elevam os valores de $\mathrm{pH}$, o que corrobora os dados obtidos por Nascimento et al. (2002) e Santos Neto (2003).

Equações de regressão múltipla foram ajustadas entre as taxas de $\mathrm{Zn}$ recuperado em função das doses adicionadas aos solos e algumas características destes que influenciam a capacidade-tampão de Zn (Quadro 8).

Os modelos de regressão ajustados para as taxas de $\mathrm{Zn}$ recuperado em função do adicionado (Quadro 8) mostram que a MO prediz $65 \%$ dos teores de $\mathrm{Zn}$ recuperados pelo Mehlich-1, enquanto o P-rem explica $49 \%$. Para o Mehlich-3, o teor de argila explica $49 \%$ e o pH $47 \%$ dos teores extraídos em função do adicionado. As taxas de recuperação de $\mathrm{Zn}$ extraído pelo DTPA são explicadas pelo $\mathrm{pH}$ em $81 \%$ e pelo $\mathrm{pH}$ e P-rem em $86 \%$.

Com base nos modelos ajustados, as variáveis de solo que melhor explicam as taxas de recuperação de Zn pelos extratores Mehlich-1 e Mehlich-3 são matéria orgânica, P-rem e teor de argila. Para o DTPA, o pH é a melhor variável explicativa das taxas de recuperação por este extrator. As taxas de recuperação de Zn para os mesmos três extratores foram mais bem explicadas pelos teores de argila e de MO (Santos Neto, 2003).

\section{CONCLUSÕES}

1. Os níveis críticos de $\mathrm{Zn}$ em solos de Cerrado, para o crescimento de mudas de eucalipto, são iguais ou inferiores a 0,23 e $0,05 \mathrm{mg} \mathrm{dm}^{-3}$ pelos extratores Mehlich-1 e DTPA, respectivamente.

2. Os teores de $\mathrm{Zn}$ no solo pelos três extratores, bem como os teores e conteúdo na planta, aumentam com a elevação das doses adicionadas de Zn e são altamente correlacionados entre si.

3. A avaliação da disponibilidade de Zn para mudas de eucalipto pode ser feita com qualquer um dos três extratores. Entretanto, o Mehlich-1 é o mais recomendado, pela facilidade operacional deste método em relação aos outros e por ele já ser usado na grande maioria dos laboratórios do País.

4. O teor de matéria orgânica e o P-remanescente são características dos solos que mais influenciam negativamente as taxas de recuperação de $\mathrm{Zn}$ pelos extratores Mehlich-1 e Mehlich-3, ao passo que, para o DTPA, as taxas de recuperação são mais influenciadas pelo $\mathrm{pH}$.

\section{LITERATURA CITADA}

ALVAREZ V., V.H. Equilíbrio de formas disponíveis de fósforo e enxofre em dois latossolos de Minas Gerais. Viçosa, MG, Universidade Federal de Viçosa, 1974. 125p. (Tese de Mestrado)

ALVAREZ V., V.H.; NOVAIS, R.F.; BARROS, N.F.; CANTARUTTI, R.B. \& LOPES, A.S. Interpretação dos resultados das análises de solos. In: RIBEIRO, A.C.; GUMARÃES, P.T.G. \& ALVAREZ V., V.H., eds. Recomendações para o uso de corretivos e fertilizantes em Minas Gerais. Viçosa, MG, CFSEMG, 1999. p.25-32.

ALVAREZ V., V.H.; NOVAIS, R.F.; DIAS, L.E. \& OLIVEIRA, J.A. Determinação e uso do fósforo remanescente. B. Inf. SBCS, 25:27-32, 2000.

BRAY, R.H. Requirements for successful soils tests. Soil Sci., 66:83-89, 1948.

CANCELA, R.C.; FREIRE, A.R.; ABREU, C.A. \& GONZÁLEZ, A.P. Eficacia de cuatro extractantes an la evaluación de la disponibilidad de cobre para maíz y soja. Bragantia, 60:205-212, 2001. 
COUTO, C.; NOVAIS, R.F.; BARROS, N.F. \& NEVES, J.C.L. Resposta do eucalipto à aplicação de zinco em amostras de solos de cerrado. R. Árvore, 9:134-148, 1985.

FAVARIN, J.L.; VITTI, G.C.; NETO, D.D.; FAVARIN Jr., J.L. \& SALGADO, P.R. Teor de zinco no café como variável da disponibilidade no solo e extrator. R. Bras. Ci. Solo, 31:1191-1196, 2007.

FERREIRA, G.B. Dinâmica das frações de micronutrientes catiônicos e esgotamento de formas disponíveis de boro, cobre, ferro, manganês e zinco, em solos de Minas Gerais. Viçosa, MG, Universidade Federal de Viçosa, 2003. 169p. (Tese de Doutorado)

FONSECA, A. F.; CAIRES, E. F. \& BARTH, G. Extraction methods and availability of micronutrients for wheat under a no-till system with a surface application of lime. Sci. Agric., 67: 60-70, 2010.

GONÇALVES Jr., A.C.; PRESTES, A.L.; RIBEIRO, O.L. \& SANTOS, A.L. Métodos extratores e fitodisponibilidade de zinco para milho em Argissolo Vermelho-Amarelo eutrófico. Sci. Agr., 7:35-40, 2006.

LINDSAY, W.L. \& NORVELL, W.A. Developemnt of a DTPA soil of zinc, iron, manganese and copper. Soil Sci. Soc. Am. J., 42:421-428, 1978.

LOPES, A.S. \& ALVAREZ V., V.H. Apresentação dos resultados das análises de solo. In: RIBEIRO, A.C.; GUIMARÃES, P.T.G. \& ALVAREZ V., V.H., eds. Recomendação para o uso de corretivos e fertilizantes em Minas Gerais. 5.ed. Viçosa, MG, CFSEMG, 1999. p.21-24.

MEHLICH, A. Determination of $\mathrm{P}, \mathrm{Ca}, \mathrm{Mg}, \mathrm{K}, \mathrm{Na}$ and $\mathrm{NH}_{4}$. Raleigh, North Carolina Soil Test Div., 1953. (Não Publicado)

MEHLICH, A. Mehlich-3 soil test extractant: A modification of Mehlich-2 extractant. Comm. Soil Sci. Plant Anal., 15:1409-1416, 1984.

MENEZES, A.A.; DIAS, L.E.; NEVES, J.C.L. \& SILVA, J.V.O. Disponibilidade de zinco para milho pelos extratores Mehlich-1, Mehlich-3 e DTPA em solos de Minas Gerais, na presença e ausência de calagem. R. Bras. Ci. Solo, 34:417-424, 2010.
NASCIMENTO, C.W.A.; FONTES, R.L.F.; NEVES, L.C.L. \& MELICIO, A.C.F.D. Fracionamento, dessorção e extração química de Zn em Latossolos. R. Bras. Ci. Solo, 26:599-606, 2002.

NOVAIS, R.F.; BARROS, N.F. \& NEVES, J.C.L. Nutrição mineral do eucalipto. In: BARROS, N.F. \& NOVAIS, R.F., eds. Relação solo-eucalipto. Viçosa, MG, Universidade Federal de Viçosa, 1990. p.25-98.

NOVAIS, R.F. \& SMYTH, T.J. Fósforo em solo e planta em condições tropicais. Viçosa, MG, Universidade Federal de Viçosa, 1999. 399p.

OBRADOR, A.; ALVAREZ, J.M.; LOPEZ-VALDIVIA, L.M.; GONZALEZ, D.; NIVILLO, M.I. \& RICO, M.I. Relationships of soil properties with $\mathrm{Mn}$ and $\mathrm{Zn}$ distribution in acid soils and their uptake by a barley crop. Geoderma, 137:432-443, 2007.

RAIJ, B. van; CANTARELlA, H.; QUAGGIO, J.A. \& FURLANI, Â.M.C. Recomendações de adubação e calagem para o Estado de São Paulo. Campinas, IAC, 1997. 285p.

RODRIGUES, F.A.V.; BARROS, N.F.; NEVES, J.C.L.; ALVAREZ V., V.H. \& NOVAIS, R.F. Disponibilidade de cobre para mudas de eucalipto em solos de Cerrado. R. Bras. Ci. Solo, 34:1923-1932, 2010.

RUIZ, H.A. Incremento da exatidão da análise granulométrica do solo por meio da coleta da suspensão (silte + argila). R. Bras. Ci. Solo, 29:297-300, 2005.

SANTOS NETO, J.A. Taxas de recuperação de zinco, cobre e boro por diferentes extratores em solos da Bahia e Minas Gerais. Viçosa, MG, Universidade Federal de Viçosa, 2003, 51p. (Tese de Mestrado)

SEQUEIRA, C.H. Disponibilidade de micronutrientes em solos e sua correlação com teores foliares em povoamentos jovens de eucalipto. Viçosa, MG, Universidade Federal de Viçosa, 2007. 36p. (Tese de Mestrado)

SEQUEIRA, C.H.; BARROS, N.F.; NEVES, J.C.L.; NOVAIS, R.F.; SILVA, I.R. \& ALLEY, M. Micronutrient soil-test levels and Eucalyptus foliar contents. Comm. Soil Sci. Plant Anal., 42:475-488, 2011.

SILVA, M.A.G.; MUNIZ, A.S.; MATA, J.D.V. \& CEGANA, A.C.V. Extratores para a quantificação do zinco e do cobre em solos cultivados com soja. Acta Sci., 26:361-366, 2003. 
R. Bras. Ci. Solo, 36:1249-1257 